\title{
Routing Algorithm of Health Monitoring Network in CPS Old Building Structure Based on Genetic Ant Colony Algorithm
}

\author{
https://doi.org/10.3991/ijoe.v12i10.6201 \\ Hongsheng Yuan, Yongjun Han \\ Lang fang Teachers University, Lang fang, Hebei, China
}

\begin{abstract}
The paper puts forward a multicast routing algorithm of genetic ant colony algorithm according to the QoS multicast routing problems existing in CPS heterogeneous network and by combining with characteristics of health monitoring on building structures. A heuristic algorithm with better time efficiency and faster solution is formed as well, which is able to provide better service quality for CPS network; HMR algorithm of balanced energy-consumption which is suitable for multilevel energy heterogeneous network is put forward so that high energy nodes and the low energy nodes can undertake more tasks of interrupted transmission. The left energy of nodes in the network is balanced, which is conducive to expanding the stability cycle of network and improving the stability of network.
\end{abstract}

Keywords-building structure health monitoring network, Genetic ant colony algorithm, CPS

\section{INTRODUCTION}

Apart from reasonable design and correct construction method, the old building safety relies more on regular repairing and maintenance after projects are completed. However, the timeliness and initiative are pervasively insufficient in regular repairing and maintenance work currently. What is more, there is a variety of damages in the structure for some large buildings, such as road and bridge, high-rise buildings, large space public buildings, etc., which are affected by all kinds of reasons, ranging from local environmental factors, fatigue of construction materials, the corrosion of metal materials to other factors exerting negative effects on the health of buildings. Moreover, the damage and material fatigue are accumulated continuously, which leads to the reduced resistance of old building structure and the degraded ability to resist all kinds of disasters [1]. When they are accumulated to some extent, the safety accidents will happen accordingly. If we are able to take appropriate monitoring measures to keep the health of the building structure and carry out real-time monitoring and testing for the health of the old building structure in the whole life cycle from the construction period to utilization period so as to diagnose whether the building structure is safe and reliable in real time, the timely, scientific and effective decision-making support for maintenance, structure reinforcement, safe usage of the old building will be made and the safety accidents of buildings will be avoided to the maximum extent accordingly.

The wireless sensor network technology under development serves for structural health monitoring on more and more buildings because of its characteristics of easy deployment, flexible application, as well as simple con- struction and maintenance. After the wireless sensor nodes are deployed reasonably, the sensor is able to collect the status information of each part of the building structure in real time so as to make it possible for acquisition of a variety of physical information sources, different types of data signals and pre-processing of data. What is more, it is also able to decompose, transfer the real time data acquired so that the necessary health parameters of the building structure are obtained according to the functional requirements of health monitoring system of building structure. Afterwards, the data collected will be transmitted to control center by means of wireless communication. The pre-set relevant diagnosis function of the control center will analyze the data monitored so as to judge whether the building is damaged and to what extent it is damaged.

The paper puts forward a multicast routing algorithm of genetic ant colony algorithm according to the QoS multicast routing problems existing in CPS heterogeneous network and by combining with characteristics of health monitoring on building structures. A heuristic algorithm with better time efficiency and faster solution is formed as well, which is able to provide better service quality for CPS network(seen as Figure 1). [2] HMR algorithm of balanced energy-consumption which is suitable for multilevel energy heterogeneous network is put forward so that high energy nodes and the low energy nodes can undertake more tasks of interrupted transmission. The left energy of nodes in the network is balanced, which is conducive to expanding the stability cycle of network and improving the stability of network.

\section{CyBER-PHYSICAL SySTEM}

Cyber-physical system is a new heterogeneous network in relation to computing, communication and control. The purposes of real-time sensing, dynamic control and information service for the physical world are realized by deep integration and distributed computing for information system and physical system. Different from the traditional Internet of things and sensor network, what CPS demands is that people are able to connect to the network whenever and wherever possible. It is integrated with personal area network (such as Bluetooth), LAN (such as Wi-Fi), mobile network (such as $2 \mathrm{G}$ and $3 \mathrm{G}$ ), satellite network, Ad Hoc network, RFID, sensor network, etc. The complexity of the network is greater than that of Internet of things and sensor networks [3].

CPS is characterized by heterogeneity, distribution and real time.It requires end-to-end QoSduringthe process of networktransmission, makes full use of the complemen- 


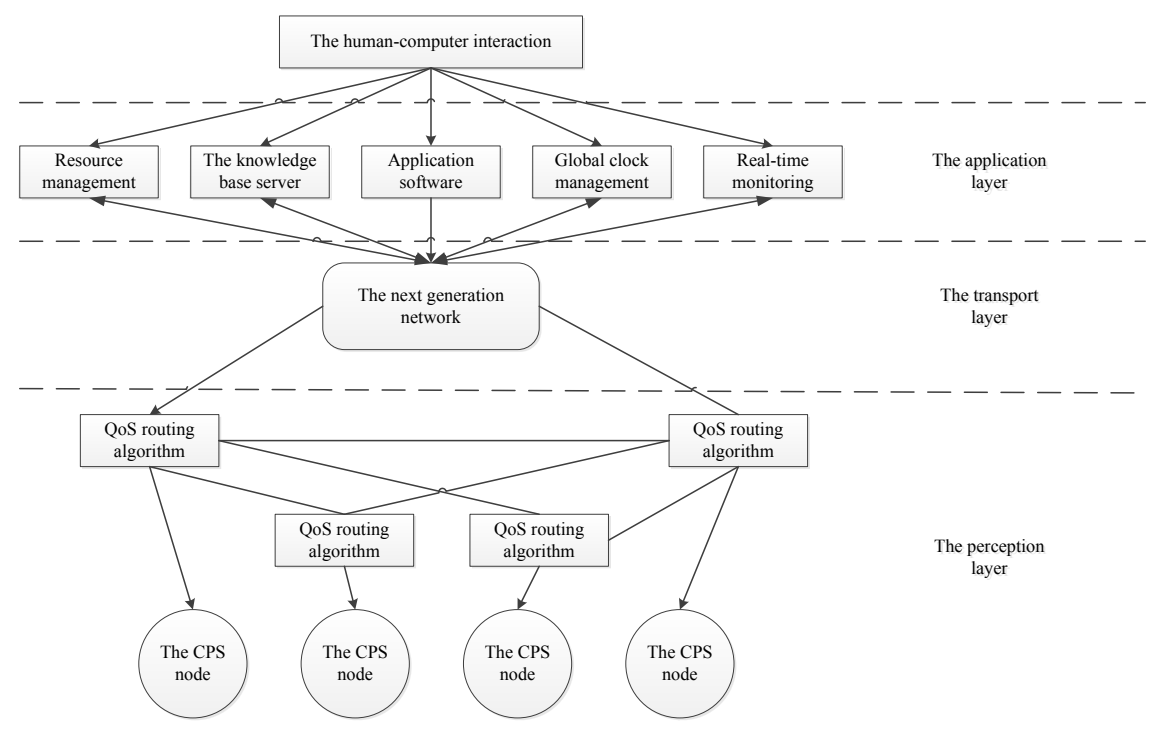

Figure 1. CPS system structure

tary characteristics of the network, and realizes the organic integration of heterogeneous network technology. Heterogeneous network integration is the inevitable development trend of the next generation network. QoS routing is one of the key technologies of multimedia information transmission for network. If it is used in CPS system, the system transmission efficiency will be guaranteed and the real-time quality of the system will be enhanced [4].

On the basis of CPS structure, the corresponding topology of wireless sensor network is designed in this chapter according to wireless sensor networks for health monitoring on building structures on the premise of considering the performance requirements of the system. It adopts the two-layer network topology structure. The scheme includes a main base station and a number of sub-networks. Each sub-network includes many sub-base stations and corresponding wireless sensor nodes. The network architecture of wireless sensor is shown in Figure 2.

In the system, every main/sub base station is consisted of one microprocessor and the wireless transceiver module. The sub base station of wireless sensor network is able to control the operation of sensor nodes in wireless sensor networks and to process the monitoring data collected by sensor nodes; while the main task of wireless sensor network nodes is to record changes of the various indicators in parts of building structure [5]. The data collected is transferred first by the sub base station and then sent to the main sub base station of wireless sensor network [6].

In the design, the main sub base station of wireless sensor network is able to directly transmit the information processed to the terminal equipment of remote users though Internet or communication satellite. In this way, the remote users can monitor the entire wireless sensor network through a variety of external networks.

III. ROUTING PROBLEMS OF HEALTH MONITORING FOR BUILDING STRUCTURE BASED ON CPS HETEROGENEOUS NETWORK

\section{A. Problem description}

In CPS network, there isa large number of network nodes. To the author's understanding, the reason is that the wired or wireless sensors are like ants, and that net- work nodeshave to send information to the information center, which is like somewhatwhere ants are able to seek food. In this process, there will be problems of network congestion, delay, etc.Therefore, how to find the optimal path in the shortest time is the research content of this section.

When analyzing the problems of routing, the network is regarded as undirected weighted graph which is usually represented by $\mathrm{P}(\mathrm{N}, \mathrm{L})$. $\mathrm{N}$ represents the network node set; $\mathrm{L}$ denotes the bidirectional link set; $s \in N$ is the node of multicast source; $M \in\{N-\{s\}\}$ is the destination set of multicast. $\mathrm{S}$ and $\mathrm{M}$ consist multicast tree $T(s, M)$, while $R_{T}(s, u)$ is the routing path for source node $\mathrm{S}$ on the multicast tree to destination $\mathrm{u}$ [7].

The main purpose of carrying out QoS routing is to determinemulticast tree with the following constraints conditions in QoS multicast routing being adhered.

Delayed constraint

$$
\operatorname{del}\left(R_{T}(s, u)\right)=\sum_{\text {leL }} \operatorname{del}(l)+\sum_{m \in N} \operatorname{del}(n) \leq D
$$

Bandwidth constraint:

$$
b w(R T(s, u))=\min \{b w(1), n \in N\} \geq B
$$

Delay jitter constraint:

$$
\begin{aligned}
& \text { del }-j i t\left(R_{T}(s, u)\right) \\
& =\sum_{l e L} d e l-j i t(1)+\sum_{n \in N} d e l-j i t(n) \leq D J
\end{aligned}
$$

Packet loss ratioconstraint:

$$
\begin{aligned}
& \operatorname{pac}-\operatorname{loss}\left(R_{T}(s, u)\right) \\
& =1-\prod_{n \in N}(1-\operatorname{pac}-\operatorname{loss}(n)) \leq P L
\end{aligned}
$$

Cost constraint: with the minimum cost when the requirements of constraint conditions in (1)-(4) are met.

$$
\cos t(T(s, M))=\sum_{l e L} \cos t(l)+\sum_{n \in N} \cos t(n)
$$

Among them, B, D, DJ, PL are respectively the bandwidth, delay, delay jitter and packet loss ratio constraints of multicast tree $T(S, M)$. 


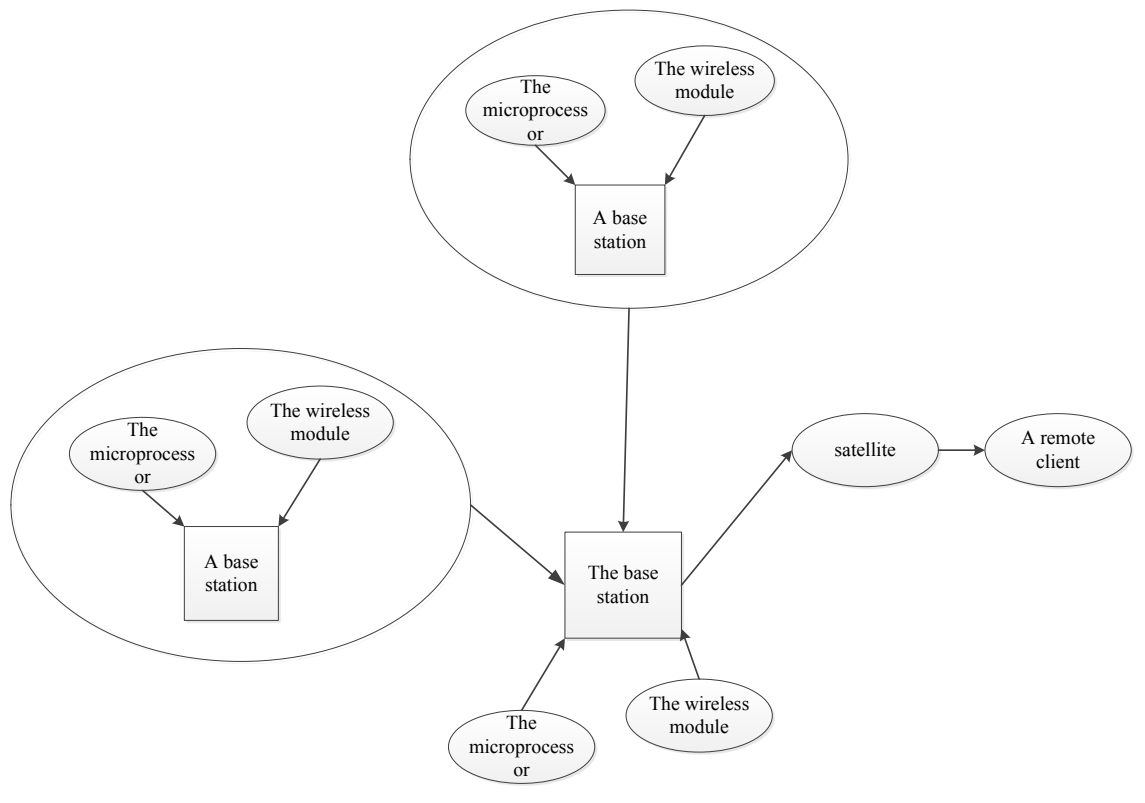

Figure 2. Wireless sensor network structure

\section{B. Rules of algorithmic}

The application of ant colony algorithmto the problem research of dynamic combinational optimization is mainly highlighted on communication network. In terms of the network routing problems, the features of ant colony algorithm agrees well with that of routing problems. Therefore, ant colony algorithm has been widely used in the network routing problem.

Every ant is actually the routing-table entry according to its experience and performance in the network just as what it does in optimization algorithm of ant colony. Ants have a unique information system which allowsthem to smell "sugar" and to gather for engorgementwith the pheromone they excrete [8]. If the path along which an ant is passing is too congested so that causing delay, there will be less pheromone left on the path and the traffic jam is mitigated. If the path is accessible or less congested, there will be more pheromone and the corresponding degree of traffic jam is leveled-up. The pheromone will volatilize as time passes, which provides real-time update onthe information system and eliminates invalid routing information immediately. As a result, theoptimum path will never appear. Even though the optimum path is congested, the sub optimum path will be found out timely. At the same time, it ensures network balance, the utilization rate and proper network load.

Slave colony optimization algorithm can take advantage of the theory ofdistributed computing with the positive feedback mechanism and greedy search being applied at the same time, which rendersthe search ability of genetic algorithm powerful. Algorithm of slave colony is a branch of the parallel computing method. However, due to the scared preliminary information, the algorithm runssuchslow that it is quite time-consumingfor the ants to converge to the optimal path. As a consequence, traffic paralysis occurseasily [9-10].

The genetic algorithm is able to search the parameters of variable coding. As for the operation of the coding, the object of the structure is operated directly, including set, sequence, matrix, trees and so on [11]. The genetic algorithm searches for a point of population instead of a single point [12]. Its parallelism is manifested in two aspects: one is that the genetic algorithm is paralleled internally to the advantage of massive parallelism; the other is the embedded parallelism of genetic algorithm which allows it to search multiple regions of the solution space by means of population at the same time with information interaction being possible in the process [13].

One important character of genetic algorithm is that it is able to search and seek the optimum one in global space by adopting probability transfer rules. Much different from those algorithms for optimization which are addressing from one path to another, this algorithm searches the optimum one in global space. In routing algorithm, The existence of plentiful redundant information in the system incurs excessive resource consumption and further renders its efficiency relatively low.

The algorithm in the paper is endowed with the advantages of slave group algorithm and genetic algorithm. Being applied in CPS system, it is able to solve the QoS routing problems, including the constraint conditions of bandwidth, delay, delay jitter, packet loss ratio and the lowest cost, so as to improve the transmission reliability and real-time performance of the system.

\section{(1) Genetic algorithm rules}

Efficiency and accuracy are taken into consideration for the genetic algorithm. The coding is compiled according to the order of traverse node.In other words, different parts of the coding that correspond to different paths are used independently to select the ID number of physical nodes which are passed on so as to carry out order compiling. Assuming that the starting point of a path is 1 and the terminating point is 8 , the coding of the path is (1 137524 8 ), which can be clearly displayed.

Apart from adopting the form of penalty function,the function of appropriate value of the algorithm takes into consideration the bandwidth, delay, delay jitter, packet loss ratio and the lowest cost:

$$
f(T(s, m))=\frac{1}{\cos t(T(s, m))}\left(A f_{d}+B f_{d j}+C f_{p l}\right)
$$



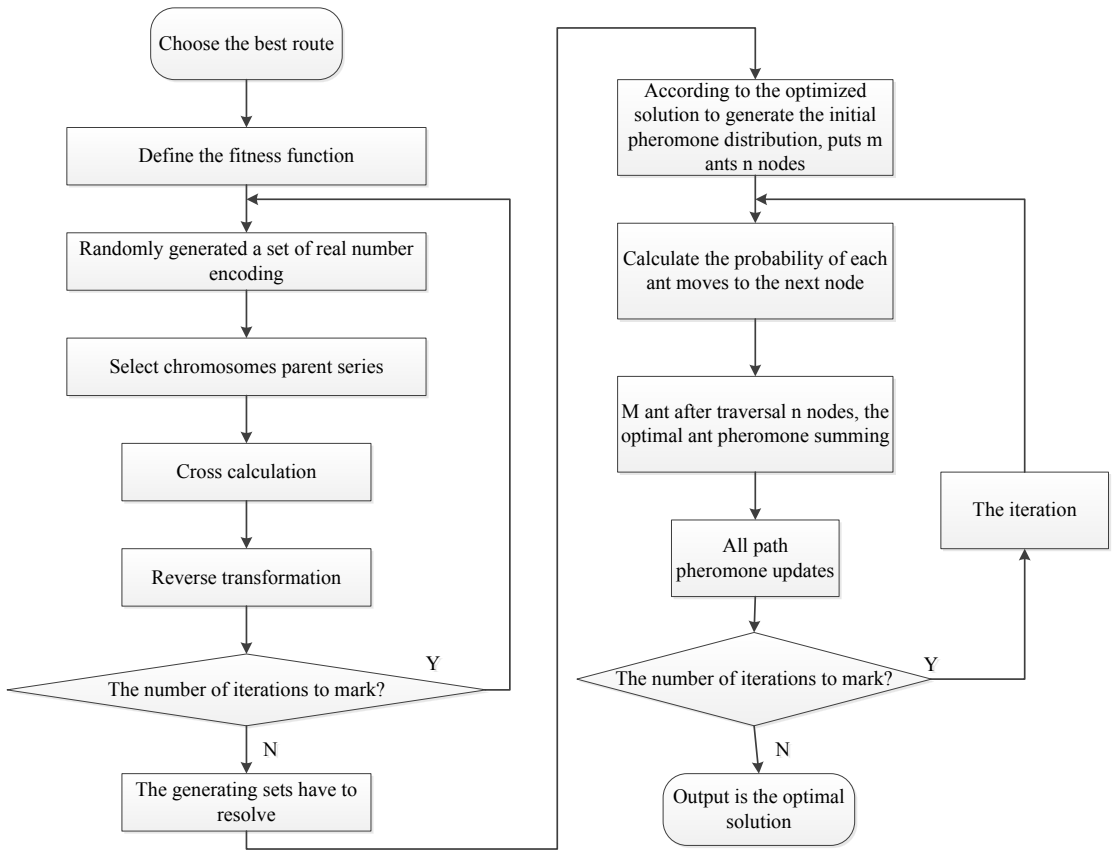

Figure 3. CAA Routing algorithm flow chart

Among them, $\mathrm{A}$ is weighted coefficient of $f_{d}, \mathrm{~B}$ is weighted coefficient of $f_{d j}$ and $\mathrm{C}$ is weighted coefficient of $f_{p l}$. They are the respective penalty functions of $\Phi_{\mathrm{d}}(Z)$, $\Phi_{\mathrm{dj}}(Z), \Phi_{\mathrm{pl}}(Z)$ - measurement delay, delay jitter, and packet loss ratio. When the routing meets the constraint conditions, the value of the function is 1 , or else the value is $r_{d}$, $r_{d j}$, or $r_{p l}$ within $(0,1)$. Their size determines the intensity of punishment. Individuals with good performance(meeting the constraint conditions) have wide adaptability while those with bad performance(not meeting the constraint conditions) adapt narrowly in comparison.

Rand function is used to generate a number of random individuals. First, the whole network will be simplified with the nodes which do not meet the constraint conditions and with the path of chain connected to them being deleted, so as to obtain the new network topology. Then a new chromosome parent string is selected. What is more, the selection strategy is carried out through fitness function.

The selection strategy for crossover operator of the algorithm is carried out by sequential crossover. The individual with the highest level of fitness selected by the algorithm is shownas follows:

In the parent string, a mating area is selected randomly and the two parent strings can be selected as

$$
\begin{aligned}
& F 1=12|3456| 789 \\
& F 2=987|6543| 21
\end{aligned}
$$

The mating area of father 2 is added before father 1 and then the mating area of father1 is added before father2.

$$
\begin{aligned}
& F 1^{`}=7654 \mid 123456789 \\
& F 2^{`}=3456 \mid 987654321
\end{aligned}
$$

Then the overlapped area of Fl' and F2' is deleted

$$
\begin{aligned}
& S 1=765412389 \\
& S 2=345698721
\end{aligned}
$$

Reverse mutation algorithm is applied as well. Assuming that there is a chromosome (6-5-4-3-2-1) which is cracked and the cracking position is within 5-4 and 3-2, reverse directional insertion can be carried out at this time with the reverse chromosome being obtained in this way. What is more, the chromosome is turned to (6-3-2-5-4-1). According to the single direction of reversal operator, the ones whose adaptive values are improved will be maintained, otherwise the reverse is invalid.

(2) Rules of ant colony algorithm

In this algorithm, the maximum and minimum MMAS algorithm of ant system is partially adopted and generates a relatively suitable algorithm accordingly.

$\square$ Calculate the transition probability

The ant will choose the next node $\mathrm{j}$ according to the current node $\mathrm{i}$

$$
j=\left\{\begin{array}{l}
\arg \max _{j \in N_{i}}\left\{\left[\tau_{i, j}(t)\right]^{\alpha}\left[\eta_{i, k}(t)\right]\right\}, q \leq q_{0} \\
j, q \leq q_{0}
\end{array}\right.
$$

Among them, $\mathrm{q}$ is arandom number that falls within the range of $(0,1)$.

$$
P_{i j}^{i}(t)=\left\{\begin{array}{l}
\frac{\tau_{i j}(t)^{\alpha}\left(\eta_{i j}\right)^{\beta}}{\sum_{u \in d_{k}} \tau_{t u}(t)^{\alpha}\left(\eta_{t j}\right)^{\beta}}, j \in d_{k} \\
0
\end{array}\right.
$$

Among them, $\tau_{i j}(t)$ pheromone trail intensity of path $(i, j)$ at time t; $\alpha$ is the relatively important $\operatorname{parameter}(\alpha \geq 0) ; \beta$ is the relatively important parameter of visibility of the path $(\beta \geq 0) ; d_{k}=\{0,1, \ldots, n-1\}$ is the number $\mathrm{k}$ sensor node set $\eta i_{j}=1 /\left(d i_{j}+d j_{i j}\right)$ which can be used in the next step. Then, the next node $\mathrm{j}$ can be selected according to the probability. 
Initial value of pheromone

The maximum and minimum MMAS algorithm of ant system sets the initial pheromone of the path as the maximum value $\tau_{\max }$ with the genetic algorithm being used to calculate the pheromone of the path. The initial value $\tau_{s}=\tau_{C}+\tau_{G}$, where $\tau_{G}$ is a constant which is the pheromone value calculated by genetic algorithm.

$\square$ Update of pheromone

The comprehensive updating strategy partially and wholly is adapted to producepartial updating so that ants are prohibited from converging to the same path. In terms of the whole updating, the pheromone on the path of historical optimal solution is updated with the purpose of rendering the search more instructive.

$\square$ Rules for partial update

$$
\begin{gathered}
\tau_{i j}(t+1)=(1-\rho) \cdot \tau_{i j}(t)+\rho \sum \Delta \tau_{i j}^{k}(t) \\
\Delta \tau_{i j}^{k}(t)=\frac{Q_{1}}{\cos (T(s, M))}
\end{gathered}
$$

Among them, $\tau_{i j}(t)$ is pheromone trail intensity of path $(i, j)$ at time t; $\Delta \tau_{i j}^{k}(t)$ is the number of pheromone per unit length trail of ant $\mathrm{k}$ in path $(i, j) ; \rho$ is the persistence of the path, $0 \leq \rho \leq 1$, with $(1-\rho)$ as the path attenuation and $Q_{l}$ as the constant.

Rules for global update

$$
\tau_{i j}(t+n)=(1-\alpha) \cdot \tau_{i j}(t)+\alpha \Delta \tau_{i j}(t)
$$

Among them, $\alpha$ is the evaporation coefficient of global pheromone. The pheromone modification $\Delta \tau_{i j}^{k}(t)$ is shown in formula $(10)$ :

$$
\Delta \tau_{i j}(t)=\left\{\begin{array}{l}
\frac{Q_{2}}{L_{b e s t}}, t+n \\
0, \text { others }
\end{array}\right.
$$

Among them, the optimal path value a is the constant.

The routing algorithm designed by the paper is endowed with the advantages of genetic algorithm and ant colony algorithm for seeking the optimum path. The flow of the algorithm is shown in Figure 3.

The iteration number of the algorithm is accumulated dynamically according to formula (11):

$$
C_{G}^{l+1}=\frac{\bar{C}(T)_{l+1}}{\left(\bar{C}(T)_{l}\right)^{p}}
$$

Among them, $\bar{C}(T)_{l}$ is the expressed population for evaluating the iteration number $P(n)$. The steps of algorithm are shown as follows:

(1)Randomly generate network topology structure including $\mathrm{N}$ nodes and the initialized network nodes:

(2)Set the parameters of genetic algorithm including delay, bandwidth, delay jitter, packet loss ratio and the cost;

(3)Analyze the QoS problems, deal with the network topology structure and initialize the population $P(0)$; tent:

(4)Genetic algorithm is realized in the following con-
While(1)

\{

Select the adaptive value and strategy. In $P(N G)$, determine the selecting strategy $p_{l}$ for each individual:

Select two individuals with the highest adaptive value which will be passed to the next generation. Among them, $\mathrm{g}$ is the iterative algebraic counter;

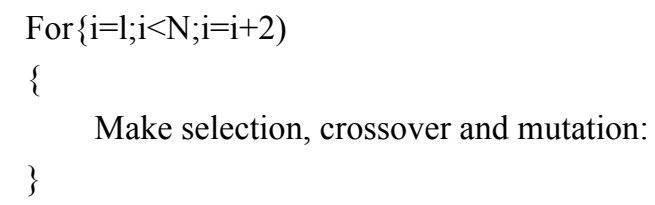

In $\mathrm{P}(\mathrm{g}+\mathrm{l})$, calculate the adaptive value of individual and the counter is added by 1 ;

If (meeting the condition of stopping)

\{break;

\}

(5)According to the results of genetic algorithm, initiate the ant colony algorithm of pheromone;

(6)Set up the parameter of ant colony algorithm

(7)The realization of ant colony algorithm is

$$
\operatorname{For}(\mathrm{j}=0 ; \mathrm{j}<\mathrm{N} ; \mathrm{j}++)
$$

\{

Initialize $\mathrm{m}$ ants in the source which is bigger than 5;

According to the strategy for path selection, every aunt will select the next node; after the next node is selected, the pheromone of the path selected will be updated according to the local updating strategy;

According to the strategy for path selection, every aunt will select the next node; after the next routing is selected, the pheromone of the path selected will be updated according to the global updating strategy;

If (meeting the condition of stopping)

\{break;

\}

\section{THE RESULTS AND ANALYSIS OF SIMULATION RESULT}

In the section, the simulation experiment is carried out for the algorithm. Firstly, a scene of simulation experiment is set up. The simulation area of the section is $1200 \mathrm{~m} \times 800 \mathrm{~m}$ in which there are 200 sensor nodes being distributed randomly. The effective area of each node is $150 \mathrm{~m}$. The node of each sensor is movable and the moving mode adapts random waypoint model. The speed scope of the node is set up as $(0,10) \mathrm{m} / \mathrm{s}$. The total number of source node and purpose nodes is 100 . And it is in line with the Poisson distribution.

The parameter of genetic algorithm is set up as: the iteration number is $200, \mathrm{pc}=0.45, \mathrm{pm}=0.05$. The parameter of ant algorithm is set up as:the number of ants is 100; the initial pheromone is 50 which is added by 2 each time with $\alpha=1, \beta=2, Q_{1}=50, Q_{2}=10, \rho=0.5, \rho_{2}=0.5, \tau_{i, j}(0)=10$.

The parameter of genetic algorithm is set up as: Network size $\mathrm{N}=200$, the range of residence time is $(0$, $1000)$, where 0 means the nodes are moving constantly and 1000 means the nodes are motionless. Finally, the algorithm is compared with the results of genetic algorithm 
and ant colony algorithm. In the simulation experiment, the residence time is the parameter related to change rate of network topology. The delaying comparison of the algorithm is shown in Figure 4. The comparison of algorithm transmission rate is shown in Figure 5. The comparison of algorithm transmission time is shown in Figure 4.

With the increasing stopping time of the experimental network, the relevant power consumption of the experimental network is constantly decreasing. From the results of delaying comparison of the algorithm shown in Figure 4 , it is found out that under the same delaying situation, the effect of genetic-ant colony algorithm is better than that of GA and AC. If the residence time of the nodes rises constantly, the topology structure of the network will become more stable and the average point-to-point delay will become smaller and smaller. Figure 5 shows the results of comparison between transmission rates for the three algorithms. Genetic-ant colony algorithm is better than the GA and AC algorithm in terms of success rate of packet transmission, which shows that genetic-ant colony algorithm has a better transmission performance than intelligent GA and AC. From the experimental results, we are able to see that compared to genetic and ant colony algorithms, the genetic-ant colony algorithm adopted in the section has a better performance. The algorithm is able to realize the effect of energy saving and to expand the life span of the network on the premise that the performance reduction of the whole wireless sensor network is limited. It is found out from the comparison results of algorithm transmission time in Figure 6 that the time for pausing of wireless sensor networks ascends constantly and the transmission time declines constantly. The system reduces the overall power consumption at the expense of losing some functional performance. The effect of genetic-ant colony algorithm is better than GA and AC algorithm. What is more, the calculation quantity of genetic-ant colony algorithm is also poorer than GA and AC algorithm with better optimized performance due to the adoption of the method of subsection processing.

\section{CONCLUSION}

The paper takes the Cyber-Physical System including perception layer, transport layer and application layer-the three layer network architecture as the basis to solve the routing problems of CPS network by adopting the geneticant colony combination algorithm and on the premise that network service quality is ensured. According to the simulation result, the optimized performance is good. Compared to the situation in which the genetic algorithm or ant colony algorithm is adapted dependently, the actual network performance after the genetic-ant algorithm is adapted in the section is better. On the premise that the performance reduction of the whole wireless sensor network is limited to some extent, the effectiveness of network energy consumption is enhanced and the life span of the network is expanded as well.

\section{REFERENCES}

[1] S.T. Cheng, "Hsu Genetic optimal deployment in wireless sensor networks", Internet Technology, vol.6(1), pp.9-18, May 2005.

[2] Y.Z. Liu, "A novel genetic algorithm for vehicle routing problem with time windows," Journal of Harbin Institute of Technology, vol. 17(3), pp.437-444, August 2010.

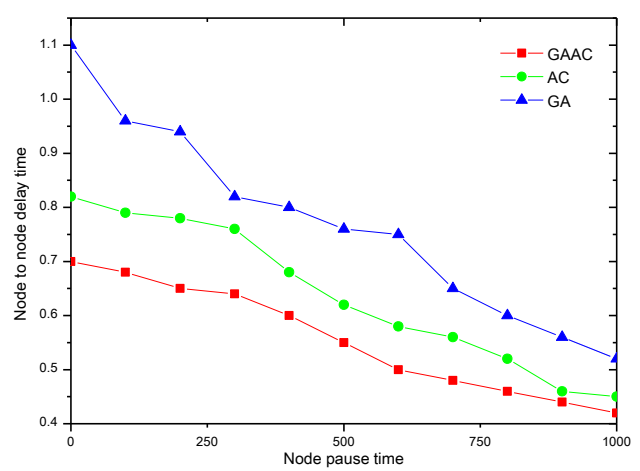

Figure 4. Delay comparison of GA,AC and GAAC algorithms

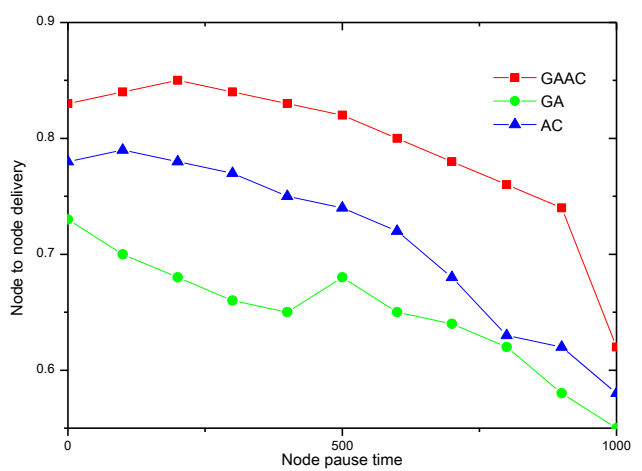

Figure 5. Note-to-node delivery ratio comparison of GA,AC and GAAC algorithms

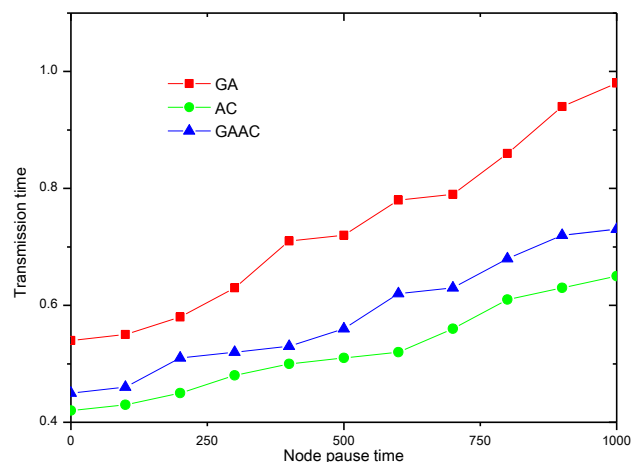

Figure 6. Transmission time comparison of GA,AC and GAAC algorithms

[3] F.Akyildiz,W. Su,Y.Sankarasubramaniam, “Wireless sensor networksra survey", Computer Networks Journal, vol. 38(4), pp. 393-422, April 2002. http://dx.doi.org/10.1016/S13891286(01)00302-4

[4] I.Nauman, A.Irfan, "Multihop clustering algorithm for load balancing in wireless sensor networks", International Journal of Simulation: Systems, Science and Technology, vol. 8(3), pp.13-25, June 2007.

[5] W.R. Heinzelman, A.P.Chandrakasan, H.BalakrishmmAn, "Application-specific protocol architecture for wireless microsensor networks", IEEE Transactions on Wireless Communications, vol. 1(4), pp.660-670, August 2002. http://dx.doi.org/10.1109/TWC. $\underline{2002.804190}$

[6] L. Qing, Q.X. Zhu, M.W. Wang. "A distributed energy-efficient clustering algorithm for heterogeneous wireless sensor networks," Journal of Software, vol.17(3), pp. 481 -489, August 2006. http://dx.doi.org/10.1360/jos170481

[7] Q. Sun, Z.Y. Yang, "A new algorithm for vertices-constrained shortest path", Computer Engineering, vol. 28(9), pp. 73-74, July 2002. 
[8] A. Bertrand, M.Moonen, "Distributed adaptive estimation of covariance matrix eigenvectors in wireless sensor networks with application to distributed PCA,"Computer Engineering, vol. 10(4), pp. 120-135, August 2014. http://dx.doi.org/10.1016/j.sigpro. 2014.03.037

[9] C. Fen. "Study of agent reliability under multi-agent cooperative decision-making", Pattern Recognition and Artificial Intelligence, vol. 21(5), pp. 621-626, May 2008.

[10] B.Gorain, P. S. Mandal. "Approximation algorithms for sweep coverage in wireless sensor networks", Journal of Parallel and Distributed Computing, vol. 74(8), pp. 2699-2707, February 2014http://dx.doi.org/10.1016/j.jpdc.2014.02.009

[11] L.L. Hung, Y.W. Huang, "Chun Cheng Lin. Temporal coverage mechanism for distinct quality of monitoring in wireless mobile sensor networks", Ad Hoc Networks, vol. 21(10), pp. 97-108, June 2014. http://dx.doi.org/10.1016/j.adhoc.2014.05.003

[12] H.Tianfield. "Multi-Agent autonomic architecture and its application in E- Medicine "Washington: IEEE Computer Society, vol. 13(2), pp.234-239, May 2003.

[13] T. Jason, S.Ferat, "Cooperation of decision-theoretic agents in the context of multi-agent systems", Washingron: Institute of Electrical and Electronics Engineers Inc, vol. 19(12), pp.224-231, May 2004.

\section{AUTHORS}

Hongsheng Yuan works in school of Architectural Engineering, Lang fang Teachers University, Lang fang, Hebei, China. His research directions are historical building protection and computer aided historical building protection (e-mail: yhs221@163.com).

Yongjun Han is an engineer and works in School of Architectural Engineering, Lang fang Teachers University, Lang fang, Hebei, China. His research direction is building construction.(e-mail: 1137802258@qq.com).

This study was supported by Lang fang Science and Technology R\&D Program 2014 (2014013103) and Science Research Project of Lang fang Teachers University (LSLY201402). Submitted 03 October 2016. Published as resubmitted by the authors 16 October 2016. 